\title{
From Theory to Evidence: Long-Term Evaluation of the Mechanism of Action and Flap Integration of Distal Vascularized Lymph Node Transfers
}

\author{
Ketan M. Patel, MD ${ }^{1}$ Chia-Yu Lin, MSc ${ }^{1}$ Ming-Huei Cheng, MD, MBA, FACS ${ }^{1}$ \\ ${ }^{1}$ Division of Reconstructive Microsurgery, Department of Plastic and \\ Reconstructive Surgery, Chang Gung Memorial Hospital, College of \\ Medicine, Chang Gung University, Taoyuan, Taiwan \\ J Reconstr Microsurg 2015;31:26-30.

\begin{abstract}
Address for correspondence Ming-Huei Cheng, MD, MBA, FACS, Division of Reconstructive Microsurgery, Department of Plastic and Reconstructive Surgery, Chang Gung Memorial Hospital, College of Medicine, Chang Gung University, 5, Fu-Hsing Street, Kweishan, Taoyuan 333, Taiwan (e-mail: minghueicheng@gmail.com).
\end{abstract}

Abstract

Keywords
- vascularized lymph
node transfer
- lymphedema
- lymphatic
microsurgery
- lymphedema surgery
- indocyanine green

Background Nonanatomic (distal) placement of vascularized lymph node (VLN) transfers have shown efficacy in the treatment of extremity lymphedema, but the mechanism by which these flaps provide relief of lymphedema remains unclear. Intrinsic lymphovenous connections have been previously shown to exist in the transferred flap. But, the long-term interaction of the VLN flap and surrounding lymphedematous extremity has not been previously investigated.

Patients and Methods A retrospective review of a prospective maintained database of patients who underwent VLN transfer was evaluated. Patients who underwent distal VLN transfer and had more than 1-year follow-up were identified. Lymphodynamic evaluation was performed using 0.3 to $0.6 \mathrm{~mL}$ indocyanine green (ICG) injection at $5 \mathrm{~cm}$ proximal to the flap edge on identified patients. Migration direction of dye and latency period was evaluated.

Results In total, 20 patients were identified who met inclusion criteria. Average longterm follow-up was 27.3 months. The average circumference reduction of the affected extremity was $40.5 \%$. ICG appearance within the VLN flap was found in all patients occurring on average in 178.3 seconds. In all cases, flow occurred in the distal direction (toward the flap) with proximal placement of dye. Latency period was found to inversely correlate with circumference reduction $(p<0.01)$.

Conclusions Distal, nonanatomic placement of VLN flaps provide sustained limb circumference reduction in extremity lymphedema patients following a minimum of 1 -year postoperatively. Flap integration with the recipient site reliably occurs as witnessed with consistent ICG drainage, and occurs in the gravity-dependent direction. Faster clearance of ICG will result in improved clinical limb circumference reduction.
Surgical treatment of lymphedema utilizing vascularized lymph node (VLN) transfer has becoming increasingly popular in recent years. Despite its popularity, the mechanism by which VLNs provide relief for symptomatic obstructive lymphedema is poorly understood. The most commonly regarded theory is based on the induction of lymphangiogenesis and

received

February 4, 2014

accepted after revision

April 8, 2014

published online

August 19, 2014 reconstitution of lymphatic channels. Animal studies have described the basis for this theory, ${ }^{1-4}$ which has prompted clinicians to use VLN flaps to treat obstructive lymphedema. ${ }^{5-8}$

Anatomic (proximal) placement of VLNs is a popular recipient site and is likely based on the theory of local lymphangiogenesis and reconstitution of normal lymphatic
Copyright $\odot 2015$ by Thieme Medical Publishers, Inc., 333 Seventh Avenue, New York, NY 10001, USA. Tel: +1(212) 584-4662.
DOI http://dx.doi.org/ 10.1055/s-0034-1381957. ISSN 0743-684X. 
flow. In addition to anatomic placement of VLNs, our center and others have previously published on nonanatomic (distal) placement of VLN flaps for obstructive lymphedema., 7-13 Despite studies reporting clinical success, the mechanism by which nonanatomic placement of VLNs improve lymphedema is far less understood. We have previously proposed the concept that the main mechanism of action is based on intrinsic lymphovenous connections within the VLN, which provides lymphovenous shunting at the level of the flap. ${ }^{13}$ Indocyanine green (ICG) venous clearance can be witnessed at the time of flap inset with injection at the flap periphery, thus validating the presence of these intrinsic lymphovenous connections. ${ }^{7,13}$ Despite the proven presence of this shunting mechanism, long-term evaluation of these intrinsic connections and the interaction of the flap and surrounding lymphedematous tissue have not been evaluated. To better understand the process of lymphatic drainage following these procedures, we investigated lymphatic flow patterns in patients who have previously undergone distal, nonanatomic VLN transfers.

\section{Patients and Methods}

\section{Study Design}

An institutional review board-approved review of a prospectively maintained database was performed at Chang Gung Memorial Hospital in Linkou, Taiwan. All patients who underwent VLN flap transfers for symptomatic obstructive upper and lower extremity lymphedema between 2008 and 2012 were identified. Patients were selected for inclusion in the study if the patient (1) had follow-up more than 12 months, and (2) had distal, nonanatomic placement of a VLN flap. Patients' demographic, surgical treatment, and outcomes were evaluated. Patients were excluded if additional surgery related to the affected extremity was performed before ICG evaluation.

Circumference reduction was evaluated at standardized office visits. In the postoperative period, patients were instructed to eliminate compression therapy if they were previously using compression. In addition, measurements before revision surgery were used for the evaluation of changes related to VLN transfer and calculation of correlations.

\section{Lymphodynamic Evaluation}

In patients selected for evaluation, subdermal ICG injections (0.3-0.6 mL) were performed proximal to the VLN flap with the patient lying in the supine position. A custom-made device activated infrared signal $760 \mathrm{~nm}$ and integrated with a camcorder (Sony HD Handycam CM05; Sony Corp., Tokyo, Japan) filtering out wavelengths below $820 \mathrm{~nm}$ was used for real-time evaluation of lymphatic flow. An injection site $5 \mathrm{~cm}$ proximal to the flap edge was performed. Flow directionality and the time to appearance of the ICG dye within the flap (latency period) were specifically evaluated. Time zero was calculated following the first injection of ICG dye proximal to the flap. The appearance of dye within the subdermal lymphatic system of the flap marked the second time point used to calculate the latency period. Reported latest follow-up time and latest circumference reduction rates are represented to demonstrate long-term follow-up results. Statistical analysis using Pearson correlation was performed to investigate the relationship of flow characteristics to clinical outcomes.

\section{Results}

A total of 20 patients were identified for study inclusion (-Table 1). The average patient age and body mass index were 54.9 years and $27.1 \mathrm{~kg} / \mathrm{m}^{2}$, respectively. The average follow-up time to lymphodynamic evaluation was 27.3 months (range, 12-128 months). Of the 20 patients, 13 (65\%) had received VLN flaps for breast cancer-related lymphedema, whereas 7 patients (35\%) had treatment for postsurgical lower extremity lymphedema. The average duration of symptoms before VLN transfer was 51.2 months. Distal, nonanatomic recipient sites included the wrist (55\%) and elbow (10\%) in upper extremity lymphedema, and the ankle (35\%) in lower extremity cases. Groin (9 patients) and submental (11 patients) VLN flaps were used for all procedures. At long-term evaluation, the average circumference reduction of the affected limb was $40.5 \%$.

All evaluated patients (100\%) had distal ICG migration to flap periphery and into the VLN flap ( - Video 1). The average latency period of ICG was 178.3 seconds (range, 38-420 seconds). When assessing the impact of the latency period in relation to the clinical improvement, an inverse relationship was found between this time and degree of circumference reduction in the affected extremity $(p<0.01)$.

Video 1
A patient with symptomatic left lower extremity
lymphedema previously underwent vascularized
submental lymph node flap to the distal ankle. Before
revision surgery, the patient had lymphodynamic
evaluation with indocyanine green (ICG). The patient
was lying supine during examination. Two ICG
injections were placed proximal to the flap edge. The
clear migration of ICG can be seen to the flap with
fluorescence enveloping the flap. These findings
highlight flap integration in the surrounding
lymphedematous tissue.
Online content including video sequences viewable
at: www.thieme-connect.com/products/ejournals/
html/10.1055/s-0034-1381957.

\section{Case Example}

A 53-year-old female patient had a history of a modified radical mastectomy, axillary lymph node dissection, and postoperative radiotherapy 6 years before evaluation (-Fig. 1). She developed symptomatic left upper extremity lymphedema (Stage IV) and underwent submental VLN transfer to the volar distal forearm (- Fig. 2). Postoperatively, 


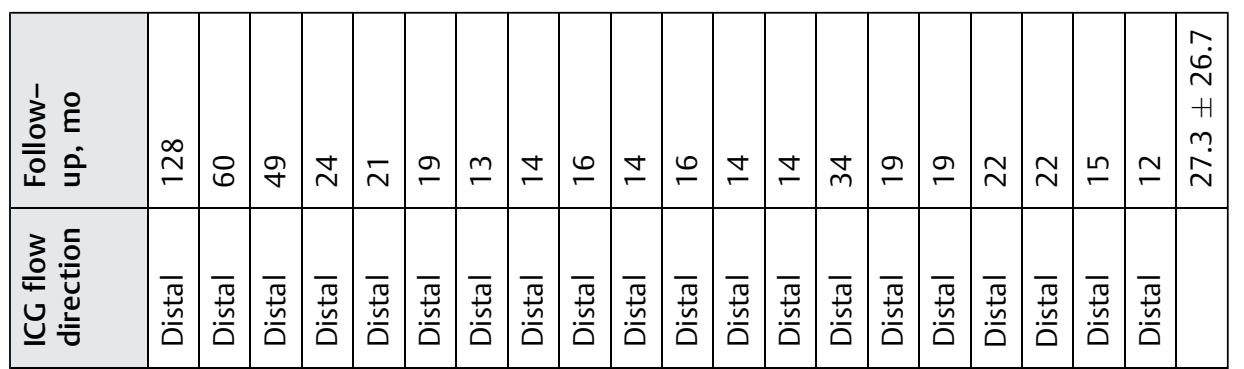

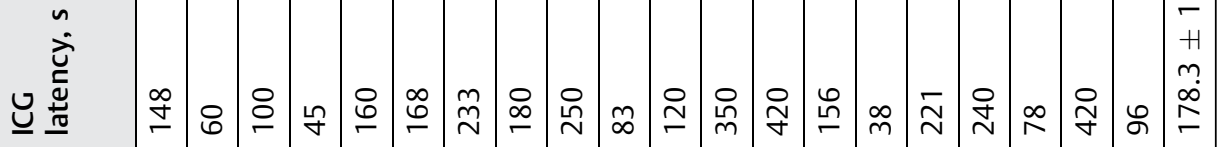

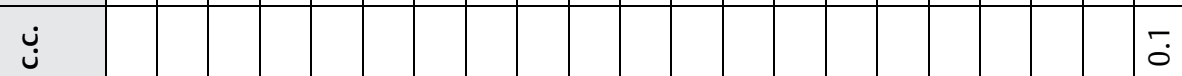

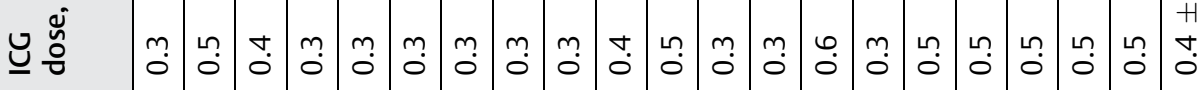
苂

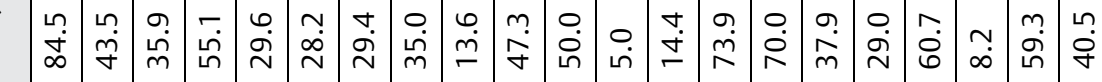

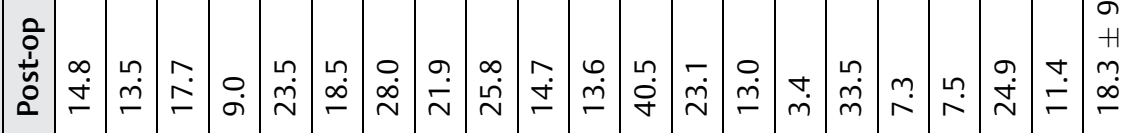

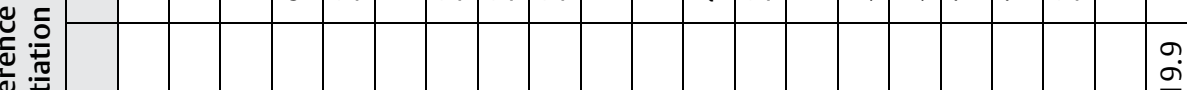
$\tilde{g}$

\begin{tabular}{|c|c|c|c|c|c|c|c|c|c|c|c|c|c|c|c|c|c|c|}
\hline 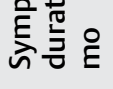 & $N$ & ஜ & $\stackrel{\varphi}{m}$ & 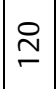 & $\stackrel{\Xi}{\sim}$ & $\stackrel{\varphi}{m}$ & $\stackrel{\unlhd}{\sim}$ & $\stackrel{\infty}{+}$ & $\stackrel{\infty}{\sim}$ & $\infty$ & $=8$ & m & $\infty$ & 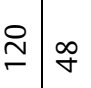 & 8 & 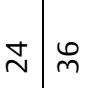 & $\simeq$ & ñ \\
\hline$\stackrel{\sum_{\infty}^{N}}{\stackrel{N}{E}}$ & $\frac{10}{\dot{m}}$ & 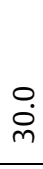 & $\begin{array}{l}N \\
\dot{m} \\
\dot{m}\end{array}$ & $\begin{array}{l}\stackrel{n}{\sim} \\
\stackrel{\sim}{\sim}\end{array}$ & $\begin{array}{l}0 \\
m \\
m \\
m\end{array}$ & $\begin{array}{l}\stackrel{9}{\dot{j}} \\
\stackrel{\sim}{*}\end{array}$ & $\begin{array}{l}0 \\
\underset{\sim}{N}\end{array}$ & $\begin{array}{l}\stackrel{\dot{\sim}}{\sim} \\
\text { a }\end{array}$ & $\begin{array}{l}\stackrel{0}{\mathrm{~N}} \\
\overrightarrow{\mathrm{N}}\end{array}$ & 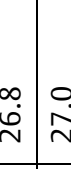 & $\begin{array}{l}\dot{v} \\
\dot{v} \\
\stackrel{\dot{\dot{d}}}{\sim}\end{array}$ & 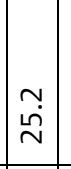 & $\stackrel{\circ}{\stackrel{\mathrm{N}}{\mathrm{N}}}$ & \begin{tabular}{l|l}
0 & 0 \\
$\dot{0}$ & $\stackrel{i}{N}$ \\
\end{tabular} & \begin{tabular}{|c|} 
\\
$\dot{i}$ \\
$m$
\end{tabular} & 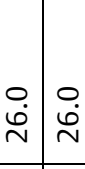 & $\bar{m}$ & $\begin{array}{l}H \\
\dot{\pi} \\
\stackrel{\sim}{\sim}\end{array}$ \\
\hline $\begin{array}{l}\overrightarrow{0} \\
\dot{g}\end{array}$ & $\tilde{6}$ & 缉 & $\widetilde{\sigma}$ & $\mid \begin{array}{l}6 \\
\end{array}$ & ठ & f & $\stackrel{q}{q}$ & in & \& & \begin{tabular}{l|l}
$n$ & $\infty$ \\
nn & in
\end{tabular} & \begin{tabular}{l|l}
$\infty$ & L \\
\end{tabular} & 6 & $\hat{\sigma}$ & 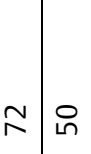 & 8 & б. & $\mathscr{f}$ & 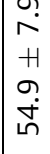 \\
\hline ف & - & $N$ & $m$ & $\nabla$ & เก & 0 & $r$ & $\infty$ & $a$ & $\div-7$ & $=1 \simeq$ & $\stackrel{m}{\square}$ & $\underset{\nabla}{\nabla}$ & $\stackrel{1}{\stackrel{1}{-}}$ & $\neq$ & $\stackrel{\infty}{\square} \stackrel{๑}{\square}$ & $\stackrel{\sim}{\sim}$ & $\bar{\alpha}$ \\
\hline
\end{tabular}

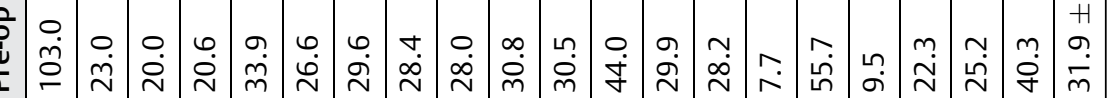

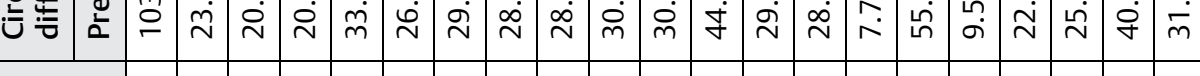

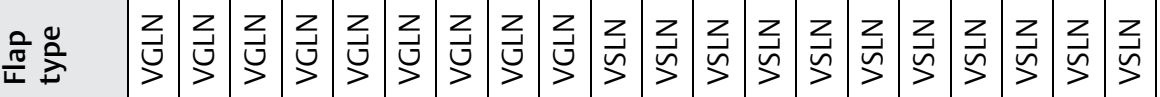
节 은든

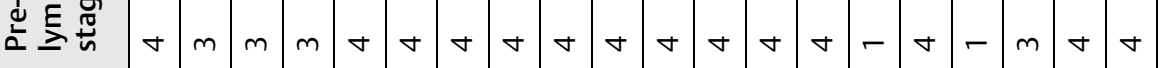

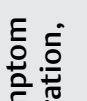




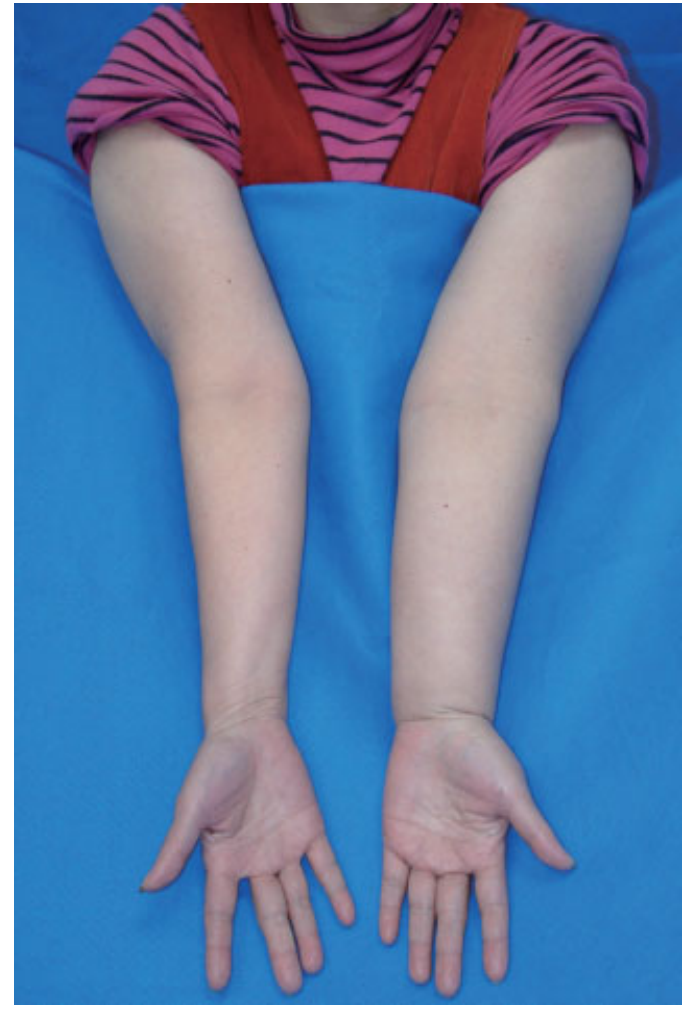

Fig. 1 A patient with symptomatic left upper extremity lymphedema is shown.

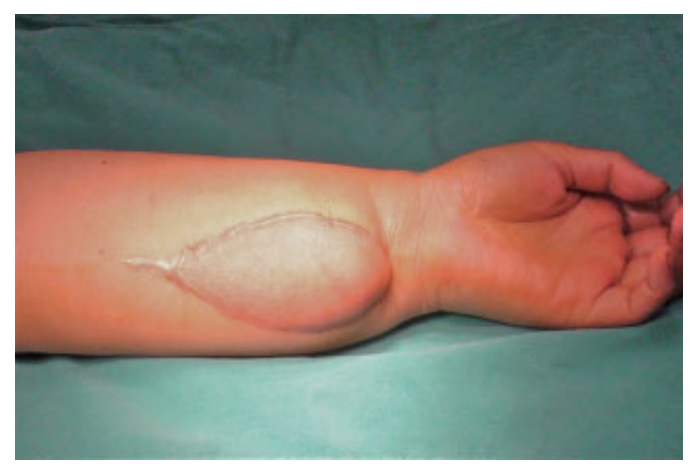

Fig. 2 The recipient site of the vascularized lymph node transfer is shown 1-year postoperatively.

she had limb circumference reduction of $47.3 \%$ over the course of 14 months ( - Fig. 3). She underwent ICG lymphodynamic evaluation at 13 months, which exhibited a latency period of 83 seconds. Distal flow of proximally placed ICG was found when the patient was lying in the supine position.

\section{Discussion}

In an early animal study by Shesol et al, VLN transfer to a lymph node-depleted area resulted in restoration of lymphatic flow, whereas transferred nodes to a normal, unoperated area did not induce additional lymphangiogenesis. ${ }^{4}$ Conclusions from this landmark animal study and others ${ }^{1-3,14,15}$ have supported the use of VLNs in anatomic (proximal) locations to replace lymph nodes and induce

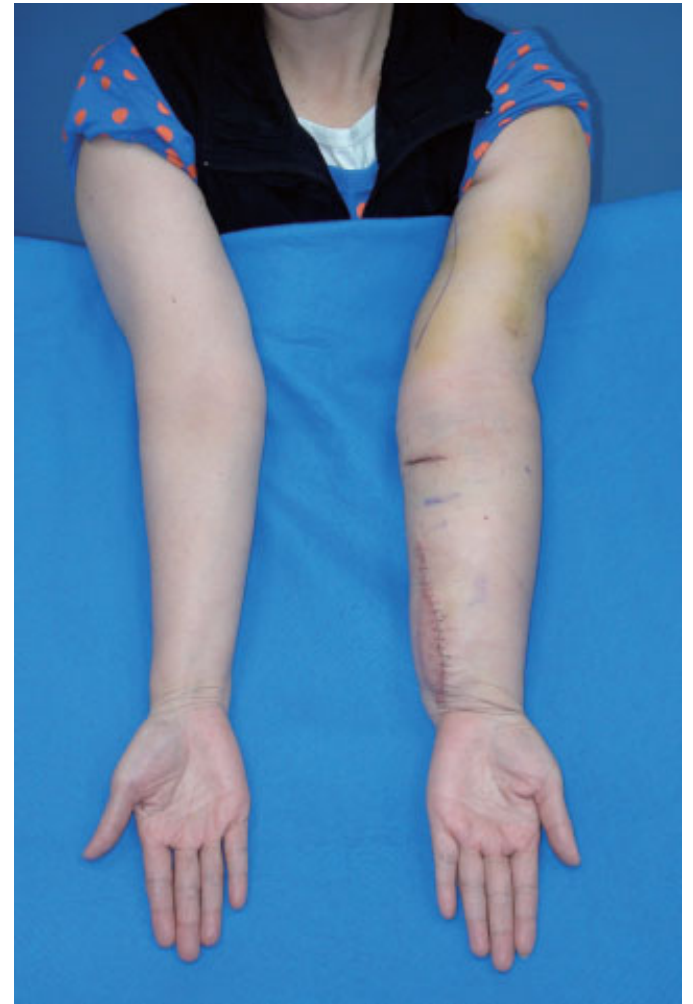

Fig. 3 The patient underwent submental vascularized lymph node transfer to the ulnar aspect of the distal forearm. She achieved approximately $47 \%$ circumference reduction to the lymphedematous extremity. The image shown is following revision surgery to the flap skin paddle and liposuction to the upper arm.

lymphangiogenesis for obstructive lymphedema with restoration of normal lymphatic flow. ${ }^{5,6}$

Unfortunately, the "stop-cock" theory may oversimplify this complex disease process and may not account for the progressive changes seen in a lymphedematous extremity. ${ }^{16}$ In the same landmark study by Shesol et al, the results suggest a significant decrease in efficacy of anatomic lymphatic restoration when VLN flap techniques are delayed from surgical lymphadenectomy. These findings highlight the temporal changes occurring to the severed ends of the lymphatic ducts, which likely result in significant scarring. ${ }^{4}$

A recent histologic evaluation found progressive and characteristic changes to the distal lymphatic collecting ducts, which correlated with decreased lymphatic function and worsening clinical lymphedema following proximal injury. ${ }^{17,18}$ Intrinsic changes to the lymphatic vasculature occur with loss of contractility ${ }^{19}$ and alterations to the secondary valve system. ${ }^{20}$ Altogether, the progressive changes that occur following proximal injury result in distal lymphatic pump failure.

Lymphatic pump failure results in backflow into lymphatic capillaries, lymphatic precollectors, and the interstitium. In addition, intrinsic changes to lymphatic collectors result in secondary valve regurgitation and bidirectional lymphatic flow. ${ }^{20,21}$ Clinically, these changes manifest as pitting edema and are consistently more pronounced in the gravity-dependent (distal) portion of the extremity. With these concepts in mind, distal nonanatomic placement of VLN flaps may represent the ideal recipient location when contemplating 
treatment for advanced lymphedema. With the results of this study, flap integration into the surrounding tissue results in ICG appearance within the flap, which confirms neo-lymphatic development. Distal flow of lymph fluid is clearly seen despite patients being in a gravity-neutral position (supine). Flow in the gravity-dependent position is amplified when patients are in the upright or standing position. Also, the latency period appears to have an inverse relationship to the degree of limb circumference reduction, which may provide clinical validation of the importance of ICG clearance via the VLN transfer. Future studies are needed to investigate why decreased latency periods are witnessed in certain patients, and why some patient cohorts have greater clinical responses as compared with matched cohorts. In addition, our findings demonstrate the continued patency of intrinsic lymphovenous connections within the VLN flap and continued venous clearance of interstitial fluid in a long-term follow-up evaluation.

There are limitations to this study that must be considered when evaluating the demonstrated outcomes. Correlations made related to the latency period may likely be influenced by factors related to the area and location of injection. Although each patient presented with similar conditions, the differences in etiology and previous treatments likely impact the results of VLN transfer techniques. Also, postoperative exercise and activity levels greatly differ between patients and likely influence perceived outcomes during long-term evaluation. Altogether, patient variables represent confounding factors during long-term evaluation of circumferential changes in lymphedema patients.

\section{Conclusions}

Distal VLN transfers provide continued venous shunting of lymphatic fluid following long-term evaluation. Recipient site integration occurs with development of new lymphatic connections. Lymphodynamic assessment with ICG demonstrates distal migration of dye toward the flap and drainage via intrinsic lymphovenous connections occurring in all patients. Decreases in the latency period may result in improvements in clinical limb circumference.

\section{Disclosures}

No funding was utilized for the preparation of this article.

\section{References}

1 Can J, Cai R, Li S, Zhang D. Experimental study of lymph node autotransplantation in rats. Chin Med J (Engl) 1998;111(3):239-241

2 Lähteenvuo M, Honkonen K, Tervala T, et al. Growth factor therapy and autologous lymph node transfer in lymphedema. Circulation 2011;123(6):613-620

3 Rabson JA, Geyer SJ, Levine G, Swartz WM, Futrell JW. Tumor immunity in rat lymph nodes following transplantation. Ann Surg 1982;196(1):92-99

4 Shesol BF, Nakashima R, Alavi A, Hamilton RW. Successful lymph node transplantation in rats, with restoration of lymphatic function. Plast Reconstr Surg 1979;63(6):817-823
5 Becker C, Assouad J, Riquet M, Hidden G. Postmastectomy lymphedema: long-term results following microsurgical lymph node transplantation. Ann Surg 2006;243(3):313-315

6 Saaristo AM, Niemi TS, Viitanen TP, Tervala TV, Hartiala P, Suominen EA. Microvascular breast reconstruction and lymph node transfer for postmastectomy lymphedema patients. Ann Surg 2012;255(3):468-473

7 Cheng MH, Chen SC, Henry SL, Tan BK, Lin MC, Huang JJ. Vascularized groin lymph node flap transfer for postmastectomy upper limb lymphedema: flap anatomy, recipient sites, and outcomes. Plast Reconstr Surg 2013;131(6):1286-1298

8 Sapountzis S, Singhal D, Rashid A, Ciudad P, Meo D, Chen HC. Lymph node flap based on the right transverse cervical artery as a donor site for lymph node transfer. Ann Plast Surg 2013. e-pub ahead of print DOI: 10.1097/SAP.0b013e31827fb39e

9 Lin CH, Ali R, Chen SC, et al. Vascularized groin lymph node transfer using the wrist as a recipient site for management of postmastectomy upper extremity lymphedema. Plast Reconstr Surg 2009; 123(4):1265-1275

10 Gharb BB, Rampazzo A, Spanio di Spilimbergo S, Xu ES, Chung KP, Chen HC. Vascularized lymph node transfer based on the hilar perforators improves the outcome in upper limb lymphedema. Ann Plast Surg 2011;67(6):589-593

11 Cheng MH, Huang JJ, Nguyen DH, et al. A novel approach to the treatment of lower extremity lymphedema by transferring a vascularized submental lymph node flap to the ankle. Gynecol Oncol 2012;126(1):93-98

12 Althubaiti GA, Crosby MA, Chang DW. Vascularized supraclavicular lymph node transfer for lower extremity lymphedema treatment. Plast Reconstr Surg 2013;131(1):133e-135e

13 Cheng MH, Huang JJ, Wu CW, et al. The mechanism of vascularized lymph node transfer for lymphedema: natural lymphaticovenous drainage. Plast Reconstr Surg 2014;133(2):192e-198e

14 Chen HC, O'Brien BM, Rogers IW, Pribaz JJ, Eaton CJ. Lymph node transfer for the treatment of obstructive lymphoedema in the canine model. Br J Plast Surg 1990;43(5):578-586

15 Tobbia D, Semple J, Baker A, Dumont D, Johnston M. Experimental assessment of autologous lymph node transplantation as treatment of postsurgical lymphedema. Plast Reconstr Surg 2009; 124(3):777-786

16 Stanton AW, Modi S, Mellor RH, Levick JR, Mortimer PS. Recent advances in breast cancer-related lymphedema of the arm: lymphatic pump failure and predisposing factors. Lymphat Res Biol 2009;7(1):29-45

17 Mihara M, Hara H, Hayashi Y, et al. Pathological steps of cancerrelated lymphedema: histological changes in the collecting lymphatic vessels after lymphadenectomy. PLoS ONE 2012;7(7): e41126

18 Hara H, Mihara M, Seki Y, Todokoro T, Iida T, Koshima I. Comparison of indocyanine green lymphographic findings with the conditions of collecting lymphatic vessels of limbs in patients with lymphedema. Plast Reconstr Surg 2013;132(6): 1612-1618

19 Modi S, Stanton AW, Svensson WE, Peters AM, Mortimer PS, Levick JR. Human lymphatic pumping measured in healthy and lymphoedematous arms by lymphatic congestion lymphoscintigraphy. J Physiol 2007;583(Pt 1):271-285

20 Suami H, Pan WR, Taylor GI. Changes in the lymph structure of the upper limb after axillary dissection: radiographic and anatomical study in a human cadaver. Plast Reconstr Surg 2007;120(4): 982-991

21 Mihara M, Hara H, Iida T, et al. Antegrade and retrograde lymphatico-venous anastomosis for cancer-related lymphedema with lymphatic valve dysfuction and lymphatic varix. Microsurgery 2012;32(7):580-584 